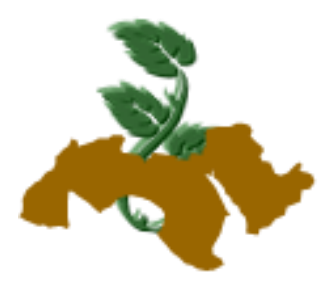

601

Arab Univ.

J. Agric. Sci., Ain Shams Univ., Cairo, 23(2), 601 - 614, 2015

\title{
COMPARATIVE MORPHOLOGICAL STUDIES ON ACHENE OF SOME TAXA OF ASTERACEAE
}

\author{
Dalia G.I. Gabr \\ Department of Botany and Microbiology, Faculty of Science (Girls Branch), Al-Azhar \\ University, Cairo, Egypt \\ E-mail: Dalia goda@hotmail.com
}

Keywords: Asteraceae, achene morphology, pappus, SEM

\begin{abstract}
Morphological structure and different characters of achene is most important from the taxonomic point of view. In present study achene shape, colour, size, texture and pappus type were recorded for 20 taxa of Asteraceae by using light microscope (LM) and scanning electron microscope (SEM). Examination of pappus by SEM recorded 6 groups; (1- Scarbous barbellate fine bristles and scarbous barbellate bristles, 2- Scarbous subulate scales free, 3- Scarious scales, 4- Paleaceous scales corona, 5- Plumose bristles and 6- Capillary barbellate). Also eight patterns were recognized based on surface sculpturing pattern: reticulate, with two subtypes, tuberculate, sulcate, puncticulate, colliculate, aculeate, lineate and striate. The data proved useful in the construction of a dichotomous indented key to the studied taxa. Twenty-two characters with 85 characters stats were used to generate anatomical key using the DELTA key-generating programs.
\end{abstract}

\section{INTRODUCTION}

Asteraceae (Compositae) is one of the largest Angiosperm families, comprises of 1590 genera and around 23,600 known species (Bremer \& Jansen, 1992 and Bremer, 1994). It is cosmopolitan in distribution, occurring in all continents except Antarctica (Hickey \& King, 1997).

(Received 16 June, 2015)

(Accepted 8 July, 2015)
The subfamilial classification had minor revisions; Cronquist (1955) recognized two subfamily and 10 tribes; (Thorne, 1983) classified the family into two subfamily and14 tribe, while (Bremer, 1994) divided it into four subfamilies and 17 tribes. More recently (Baldwin et al 2002 and Panero \& Funk, 2002) divided the family into 10 subfamilies and 35 tribes.

The fruit of Asteraceae are technically called as cypsela or achene, which develops from bicarpellary, syncarpus, epigynous, one chambered, one ovuled ovary with basal placentation. Most achen have appendage called pappus; the most recent workers have adopted the view that the pappus is a modified calyx (Cronquist, 1977; Thorne, 1983 and Takhtajan, 1997). Cypselar features are very helpful, for the separation of taxa, when flowering stage is unavailable (Jana and Mukherjee, 2012). Morphological structure and different characters of cypsela is most important from the taxonomic point of view. Also the pappus is a taxonomically important and useful organ in Asteraceae (Cassini, 1827). Cypsela or achene study has been utilized by many authors successfully for the delimitation of genera and species within the family Asteraceae as (Bhar \& Mukherjee, 2004; Chehregani \& Mahanfar, 2007; Talukdar \& Mukherjee, 2008; Jana \& Mukherjee, 2012 \& 2013; Bednorz \& Podsiedlik 2013, Jana et al 2013 and Jana \& Mukherjee, 2014). Most taxonomic affinity studies in Asteraceae (Compositae) in Egypt have focused mainly on a systematic revision as (Amin, 1978; Zareh, 1987, 1992 \& 2005; Fayed, 1987 \& 1991; Fayed and Zareh, 
1987, 1988 \& 1989; Fayed and M602ohamed, 1991a \& b; El-Karemy and Zareh, 1991; Zareh and Osman, 2004 and Zareh, 2005). Little studies other than revision are don as (Abd El-Wahid et al 2009; Abd El-Twab \& Zahran, 2010 and Osman, 2011 a \& b). The scan of achene and pappus in Asteraceae taxa growing in Egypt is not yet correctly projected.

The aim of this study was to compare the achene morphological characters for some taxa of different Asteraceae tribes to evaluate the application of this character in the classification and finding some useful characters to delimit the taxa at species level.

\section{MATERIAL AND METHODS}

The present study included achen of 20 taxa of family Asteraceae belong to three subfamily and nine tribe according to Panero \& Funk (2002), collected fresh from different localities in Egypt (Table 1). The materials studied were identified by means of comparison with specimens kept in the herbarium of the Agricultural Museum (CAIM). In addition, keys of (Tãckholm, 1974; Davis, 1975; Boulos, 2000 and Bisby et al 2010). Reference herbarium specimens of studied species were prepared and kept in the herbarium of Botany and Microbiology Department, Faculty of Science (Girls Branch) Al-Azhar University. For fruit morphology, five to seven achen were investigated to record their dimensions, shape, color, size, pappus and surface texture. Details of achen sculpture were examined by a JEOL JSM - 5500 scanning electron microscope operated at an accelerated voltage of $20 \mathrm{kv}$ and photographed. The terminology of (Bednorz \& Podsiedlik, 2013) was adopted to describe the SEM aspects of the achen coat, and the terminology of (Mukherjee and Nordenstam, 2008) for pappus structure.

\section{Numerical analysis}

Various data obtained from the description of the achene morphology and pappus were subjected to automated key generation (Key 2) using version 4.12 of the DELTA suite of program (Dallwitz et al 2000).

\section{RESULTS AND DISCUSSION}

The achene morphological characters, pappus and achene sculpture of the studied taxa are summarized in Tables 2 \& 3 and Plates 1, 2 and 3.

\section{A. Pappus}

Papuse were seen in different forms. The examination of pappus by scanning electron microscope divided the studied taxa into 6 groups:

a- Group 1- Scarbous barbellate fine bristles and scarbous barbellate bristles; it is the main group which including 12 taxa; eg. Pluchea dioscoridis \& Sonchus oleraceus (Plate 2 and Figs. 5 \& 19)

b- Group 2- Scarbous subulate scales free, including 3 taxa; eg. Carduus pycnocephalus (Plate 2 and Fig. 1)

c- Group 3- Scarious scales specific for $\mathrm{Pu}$ licaria undulate (Plate 2, Fig. 9b)

d- Group 4- Paleaceous scales corona for Anthemis melampodina subsp. deserti (Plate 2 and Fig. 10)

e- Group 5- Plumose bristles for two taxa; eg. Notobasis syriaca (Plate 2, Fig. 2)

f- Group 6- Capillary barbellate for Phagnalon rupestre (Plate 2, Fig. 7)

\section{B. Achene}

Data indicated that studied taxa were delineated on the following characteristics:

\section{Length}

Studied specimens were between 0.9 - 9 $\mathrm{mm}$. Achene lengths were different in different specimens; in Urospermum picroides it was longer than $8 \mathrm{~mm}$, in Pluchea dioscoridis less than $1 \mathrm{~mm}$ and in the reminders it was noted in a variety of sizes.

\section{Shape}

Achenes shape was also different in studied taxa. Shape of achene was Fusiform in Senecio vulgaris, Obovate in Notobasis syriaca, oblong in Centaurea aegyptiaca and Curved in Calendula arvensis. 
Table 1. Collection data of the taxa included in the present study, sub-family \& tribes according to Panero \& Funk, 2002; all localities are in Egypt.

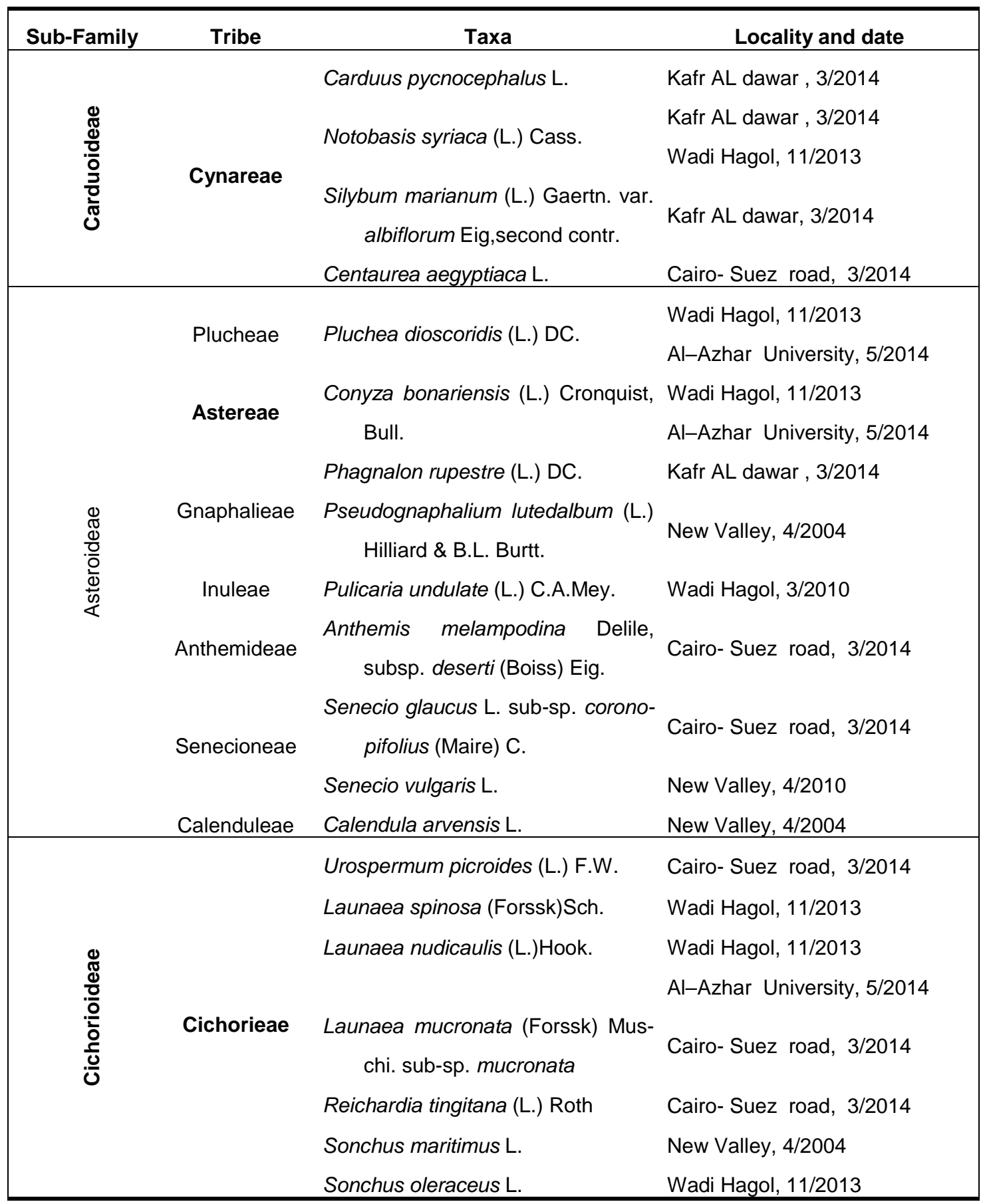




\section{Dalia Gabr}

Table 2. Comparative observations on achene morphology of the studied taxa of Asteraceae

\begin{tabular}{|c|c|c|c|c|c|c|c|c|c|c|c|c|c|c|c|c|}
\hline \multirow[b]{2}{*}{ Taxa } & \multicolumn{9}{|c|}{ Achene } & \multicolumn{7}{|c|}{ Pappus } \\
\hline & 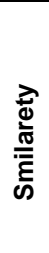 & $\frac{\grave{0}}{0}$ & $\frac{\stackrel{\Xi}{\frac{2}{d}}}{\frac{c}{\text { ஸ }}}$ & 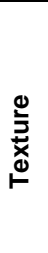 & 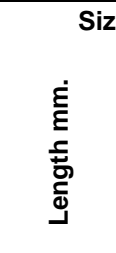 & $\begin{array}{l}\text { É } \\
\text { E } \\
\frac{c}{5} \\
\stackrel{0}{3}\end{array}$ & $\begin{array}{l}\stackrel{8}{\Phi} \\
\frac{0}{\tilde{g}}\end{array}$ & $\begin{array}{l}\text { 을 } \\
\text { 응 } \\
\text { 응 } \\
\text { के }\end{array}$ & $\begin{array}{l}\text { 离 } \\
\text { Ф̊ }\end{array}$ & 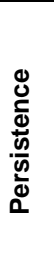 & $\frac{\grave{3}}{8}$ & 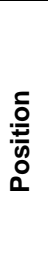 & $\begin{array}{l}\frac{1}{ \pm} \\
\text { है } \\
\text { z }\end{array}$ & 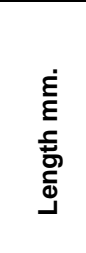 & $\stackrel{\unrhd}{\stackrel{2}{\gtrless}}$ & 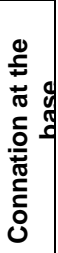 \\
\hline Carduus pycnocephalus & 1 & 2 & 1 & 1 & $4.5-5$ & 2- 2.1 & 1 & 1 & 2 & 2 & 3 & 1 & 2 & $10-15$ & 3 & 1 \\
\hline Notobasis syriaca & 1 & 1 & 2 & 1 & $5-6$ & $3-3.5$ & 2 & 2 & 2 & 2 & 1 & 2 & 2 & $12-15$ & 6 & 2 \\
\hline Silybum marianum var. albiflorum & 1 & 1 & 1 & 1 & $6-7$ & 3 & 2 & 1 & 2 & 2 & 1 & 1 & 2 & $12-14$ & 3 & 1 \\
\hline Centaurea aegyptiaca & 1 & 1 & 1 & 4 & $1.5-2$. & $0.5-1$ & 2 & 2 & 2 & 1 & 1 & 1 & 2 & $5-6$ & 3 & 2 \\
\hline Pluchea dioscoridis & 1 & 1 & 1 & 1 & $0.9-1$ & $0.1-0.2$ & 1 & 2 & 2 & 1 & 1 & 1 & 1 & $2.5-3$ & 2 & 2 \\
\hline Conyza bonariensis & 1 & 3 & 1 & 4 & $1.5-1.7$ & 0.2 & 2 & 2 & 2 & 1 & 1 & 1 & 1 & $3.2-4$ & 2 & 2 \\
\hline Phagnalon rupestre & 1 & 1 & 1 & 4 & 1 & 0.1 & 2 & 2 & 2 & 1 & 1 & 1 & 1 & 6 & 7 & 1 \\
\hline Pseudognaphalium lutedalbum & 1 & 1 & 1 & 6 & 0.5 & 0.2 & 2 & 2 & 2 & 2 & 1 & 1 & 2 & 2 & 2 & 1 \\
\hline Pulicaria undulate & 1 & 1 & 3 & 4 & $1.1-1.5$ & $0.3-0.5$ & 2 & 2 & 2 & 2 & 1 & 1 & 1 & $3-6$ & 4,2 & 1 \\
\hline Anthemis melampodina subsp. deserti & 1 & 1 & 2 & 2 & $0.9-1.9$ & 0.1 & 1 & 2 & 2 & 1 & 2 & 1 & 1 & $1.5-.8$ & 5 & 1 \\
\hline Senecio glaucus sub-sp. coronopifolius & 1 & 5 & 4 & 3 & $2-2.5$ & 0.5 & 2 & 1 & 2 & 2 & 1 & 1 & 2 & 4.5 & 1 & 2 \\
\hline Senecio vulgaris & 1 & 2 & 4 & 3 & $2.5-3$ & 0.5 & 2 & 1 & 2 & 2 & 1 & 1 & 2 & $7-9$ & 1 & 2 \\
\hline Calendula arvensis & 2 & 2 & 6 & 5 & $2.3-5$ & & 2 & 2 & 2 & 3 & - & - & - & - & - & - \\
\hline Urospermum picroides & 1 & 1 & 3 & 2 & $8-9$ & 1.5 & 1 & 1 & 1 & 2 & 1 & 1 & 2 & $1-1.2$ & 6 & 1 \\
\hline Launaea spinosa & 1 & 4 & 2 & 2 & $2.8-3$ & $1-1.2$ & 1 & 1 & 2 & 1 & 1 & 1 & 2 & $4-5$ & 1 & 2 \\
\hline Launaea nudicaulis & 1 & 4 & 2 & 1 & $1.2-1.5$ & $0.2-0.3$ & 1 & 2 & 2 & 1 & 1 & 1 & 2 & $7-9$ & 1 & 2 \\
\hline $\begin{array}{l}\text { Launaea mucronata sup-sp. mucrona- } \\
\qquad \text { ta }\end{array}$ & 1 & 1 & 4 & 2 & 3 & 0.4 & 1 & 1 & 2 & 1 & 1 & 1 & 2 & $7-8$ & 1 & 2 \\
\hline Reichardia tingitana & 1 & 1 & 5 & 2 & $1.3-1.9$ & $0.3-0.4$ & 2 & 2 & 2 & 2 & 1 & 1 & 2 & $9-10$ & 2 & 1 \\
\hline Sonchus maritimus & 1 & 4 & 1 & 2 & $2-2.3$ & $0.4-1$ & 1 & 1 & 2 & 2 & 1 & 1 & 2 & $8-9$ & 1 & 2 \\
\hline Sonchus oleraceus & 1 & 1 & 4 & 2 & $2.5-3$ & 8.1 & 1 & 1 & 2 & 2 & 1 & 1 & 2 & $5-7$ & 1 & 2 \\
\hline $\begin{array}{l}\text { Achene similarity: } 1=\text { Homogenous; } 2= \\
\text { Achene colour: } 1=\text { Brown; } 2=\text { Green; } 3= \\
\text { Achene shape: } 1=\text { Oblong; } 2=\text { Obvate; } 3 \\
\text { Achene texture: } 1=\text { Smooth; } 2=\text { Tubercu } \\
\text { Achene ridges: } 1=\text { Present; } 2=\text { Absent. } \\
\text { Achene stylopodium: } 1=\text { Conspicuous; } 2 \\
\text { Achene beak: } 1=\text { Present; } 2=\text { Absent. } \\
\text { Pappus persistence: } 1=\text { Persistent; } 2=\mathrm{D} \\
\text { Pappus colour: } 1=\text { White; } 2=\text { Yellow; } 3= \\
\text { Pappus position: } 1=\text { Terminal; } 2=\text { Sub-te } \\
\text { Pappus number: } 1=\text { Limited (less than } 1 \\
\text { Pappus types: } 1=\text { Scarbous barbellate fi } \\
\text { ious scales; } 5=\text { Paleaceous scales to for } \\
\text { Pappus connate at the base: } 1=\text { Connat }\end{array}$ & $\begin{array}{l}\text { cidu } \\
\text { lello } \\
\text { mine } \\
\text { ); } 2 \\
\text { e bri } \\
\text { n co }\end{array}$ & $\begin{array}{l}\text { us; } \\
\text { vish } \\
\text { I. } \\
\text { Unlies } \\
\text { stli }\end{array}$ & $\begin{array}{l}\text { ic. } \\
=\text { Yell } \\
\text { Obva } \\
\text { airy; } \\
\text { icuou } \\
3=\text { At } \\
\text { brow } \\
\text { imitec } \\
2=\subseteq \\
6=P\end{array}$ & $\begin{array}{l}4=S \\
\text { us. } \\
\text { bser } \\
\text { in. } \\
\text { d (m } \\
\text { Scar }\end{array}$ & $\begin{array}{l}\text { sh green; } \\
4=\text { Fusifor } \\
\text { parsely ha } \\
\text { t. } \\
\text { ore than } 1 \\
\text { bous barb } \\
\text { ose bristle }\end{array}$ & $\begin{array}{l}\text { 5). } \\
\text { ellate bri } 7=\mathrm{Ca}\end{array}$ & ; 3 & 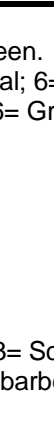 & 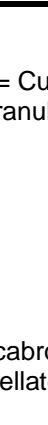 & - & & & & free & $S c$ & \\
\hline
\end{tabular}


Table 3. Comparative observations on achene coat micrography of the studied taxa of Asteraceae

\begin{tabular}{|c|c|c|c|c|c|c|}
\hline \multirow[b]{2}{*}{ Taxa } & \multirow{2}{*}{$\begin{array}{c}\text { Achene coat } \\
\text { pattern } \\
\text { sculpture }\end{array}$} & \multicolumn{3}{|c|}{ Anticinal wall } & \multicolumn{2}{|c|}{ Periclincal wall } \\
\hline & & Shape & Thicknes & Level & Level & Texture \\
\hline Carduus pycnocephalus & 1 & 1 & 1 & 1 & 1 & 1 \\
\hline Notobasis syriaca & 2 & 2 & 1 & 1 & 1 & 1 \\
\hline Silybum marianum var. albiflorum & 3 & 2 & 1 & 3 & 1 & 1 \\
\hline Centaurea aegyptiaca & 4 & 3 & 2 & 1 & 1 & 3 \\
\hline Pluchea dioscoridis & 7 & 3 & 1 & 2 & 3 & 1 \\
\hline Conyza bonariensis & 6 & 3 & 1 & 2 & 3 & 1 \\
\hline Phagnalon rupestre & 3 & 2 & 1 & 2 & 3 & 3 \\
\hline Pseudognaphalium lutedalbum & 3 & 2 & 1 & 2 & 3 & 1 \\
\hline Pulicaria undulate & 5 & 2 & 1 & 1 & 1 & 1 \\
\hline $\begin{array}{l}\text { Anthemis melampodina subsp. } \\
\text { deserti }\end{array}$ & 3 & 2 & 1 & 3 & 2 & 3 \\
\hline $\begin{array}{l}\text { Senecio glaucus sub-sp. Corono- } \\
\quad \text { pifolius }\end{array}$ & 8 & 4 & 1 & 1 & 1 & 3 \\
\hline Senecio vulgaris & 8 & 4 & 1 & 1 & 1 & 3 \\
\hline Calendula arvensis & 9 & 4 & 1 & 3 & 2 & 4 \\
\hline Urospermum picroides & 3 & 2 & 1 & 3 & 2 & 3 \\
\hline Launaea spinosa & 3 & 2 & 1 & 3 & 2 & 1 \\
\hline Launaea nudicaulis & 4 & 3 & 2 & 2 & 3 & 3 \\
\hline $\begin{array}{l}\text { Launaea mucronata sup-sp. mu- } \\
\quad \text { cronata }\end{array}$ & 8 & 4 & 1 & 1 & 1 & 2 * \\
\hline Reichardia tingitana & 8 & 4 & 1 & 1 & 1 & 3 \\
\hline Sonchus maritimus & 3 & 4 & 1 & 3 & 2 & 1 \\
\hline Sonchus oleraceus & 3 & 4 & 1 & 3 & 2 & 1 \\
\hline
\end{tabular}

Achene coat pattern sculpture: $1=$ Puncticulate; $2=$ Colliculate; $3=$ Reticulate - rugose; $4=$ Sulcate; $5=$ Aculeate; $6=$ Lineate; $7=$ Striate; $8=$ Tuberculate; $9=$ Reticulate.

Anticinal wall shape: $1=$ Round; $2=$ Undulate; $3=$ Line; $4=$ Wavy.

Anticinal wall thicknes: $1=$ Thin; $2=$ Thick.

Anticinal wall level: $1=$ Grooved; $2=$ Convex; $3=$ Raised

Periclincal wall level: $1=$ Convex; $2=$ Concave; $3=$ Grooved.

Periclincal wall texture: $1=$ Smooth; $2=$ Striated; $3=$ Warty; $4=$ Papillate.

${ }^{*}=$ Secondary roseate structure 

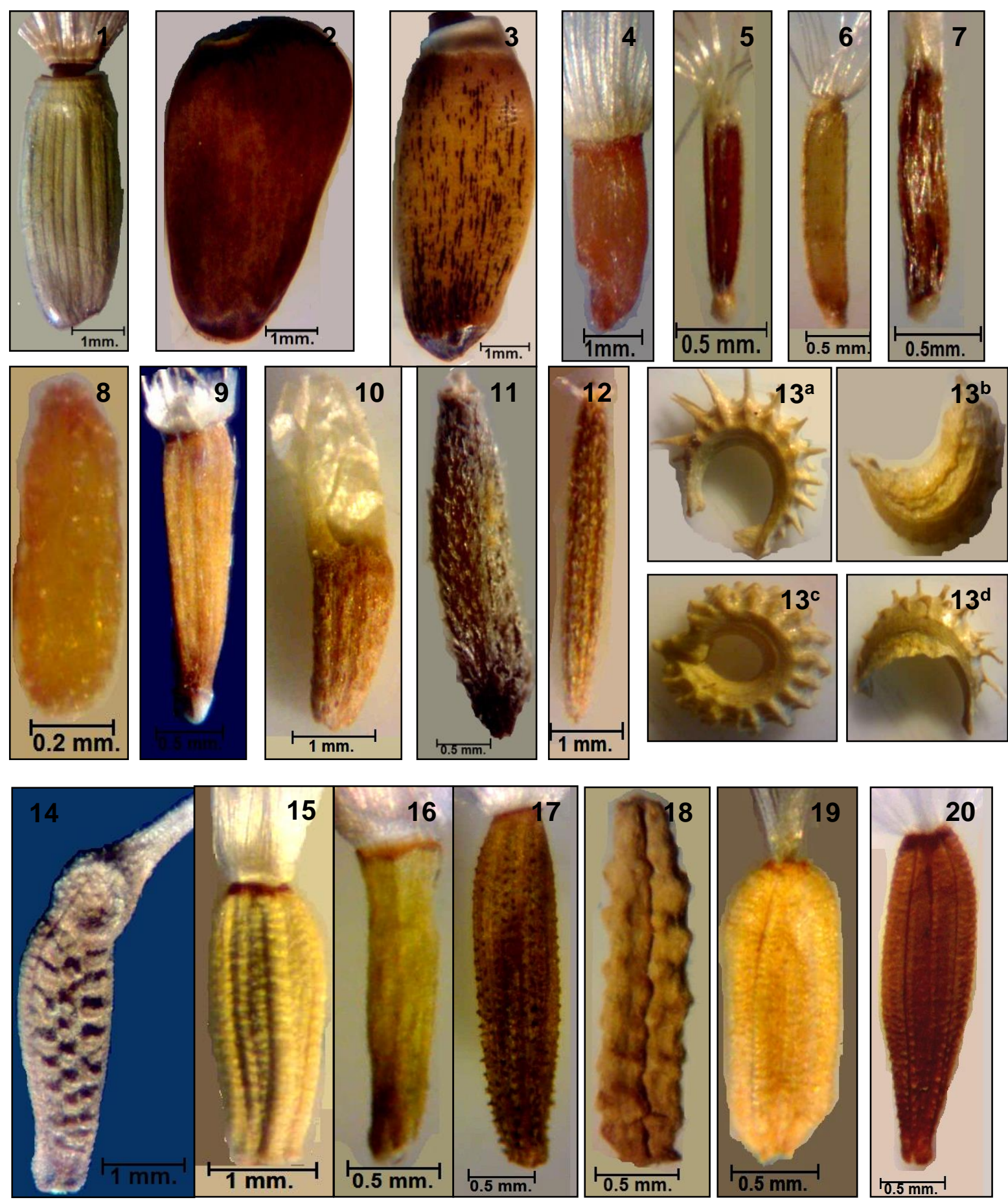

Plate 1. Achene morphology of 20 taxa of Asteraceae as revealed by light microscopy: 1. Carduus pycnocephalus; 2. Notobasis syriaca; 3. Silybum marianum var. albiflorum; 4. Centaurea aegyptiaca; 5 . Pluchea dioscoridis; 6. Conyza bonariensis; 7. Phagnalon rupestre; 8. Pseudognaphalium lutedalbum ; 9. Pulicaria undulate; 10. Anthemis melampodina subsp. deserti; 11. Senecio glaucus sub-sp. Coronopifolius; 12. Senecio vulgaris; 13. Calendula arvensis; 14 . Urospermum picroides; 15. Launaea spinosa; 16. Launaea nudicaulis; 17.Launaea mucronata sub-sp. mucronata; 18. Reichardia tingitana; 19. Sonchus maritimus; 20. Sonchus oleraceus. 

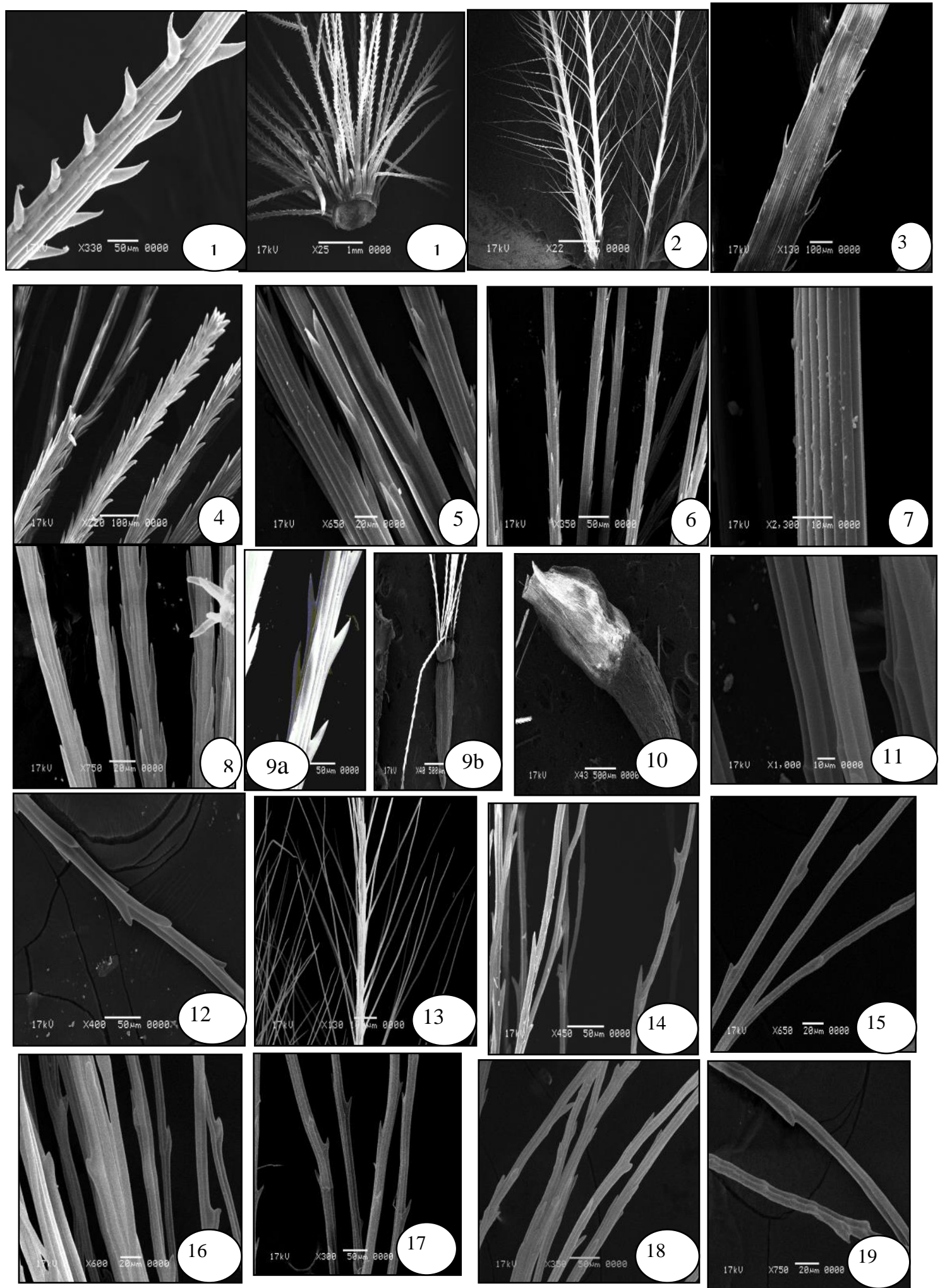

Plate 2. Pappus morphological types of 19 taxa of Asteraceae as revealed by SEM : 1. Carduus pycnocephalus; 2. Notobasis syriaca; 3. Silybum marianum var. albiflorum; 4. Centaurea aegyptiaca; 5. Pluchea dioscoridis; 6 . Conyza bonariensis; 7. Phagnalon rupestre; 8. Pseudognaphalium lutedalbum ; 9. Pulicaria undulate;10. Anthemis melampodina subsp. deserti; 11. Senecio glaucus sub-sp. Coronopifolius; 12. Senecio vulgaris; 13 . Urospermum picroides; 14. Launaea spinosa; 15. Launaea nudicaulis; 16. Launaea mucronata sub-sp. mucronata; 17. Reichardiatingitana; 18. Sonchus maritimus; 19. Sonchus oleraceus 

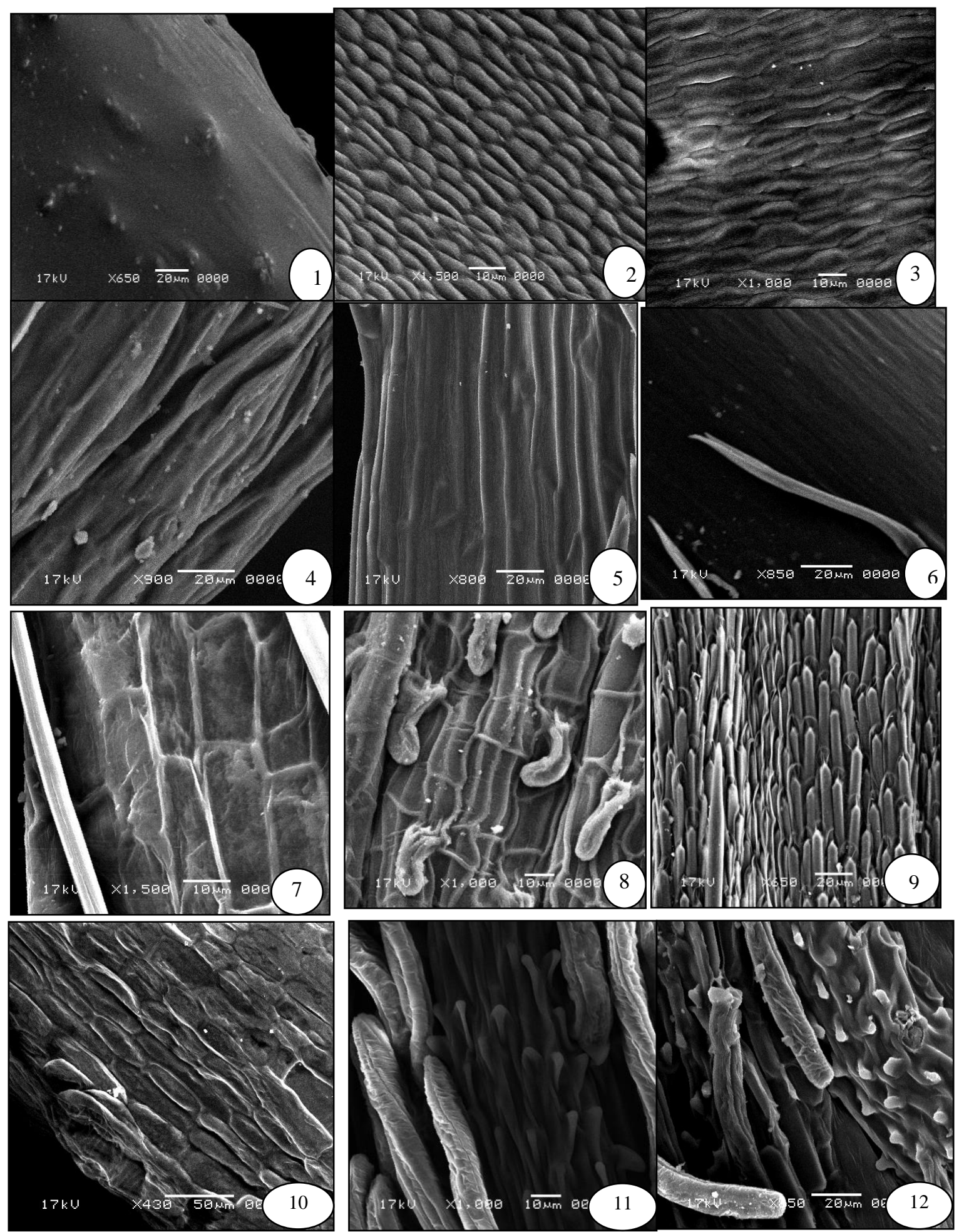

Plate 3. Achene surface sculpture for 20 taxa of Asteraceae as revealed by SEM: 1. Carduus pycnocephalus; 2. Notobasis syriaca; 3. Silybum marianum var. albiflorum; 4. Centaurea aegyptiaca; 5 . Pluchea dioscoridis; 6. Conyza bonariensis; 7. Phagnalon rupestre; 8. Pseudognaphalium lutedalbum ; 9. Pulicaria undulate; 10. Anthemis melampodina subsp. deserti; 11. Senecio glaucus sub-sp. Coronopifolius; 12 . Senecio vulgaris 

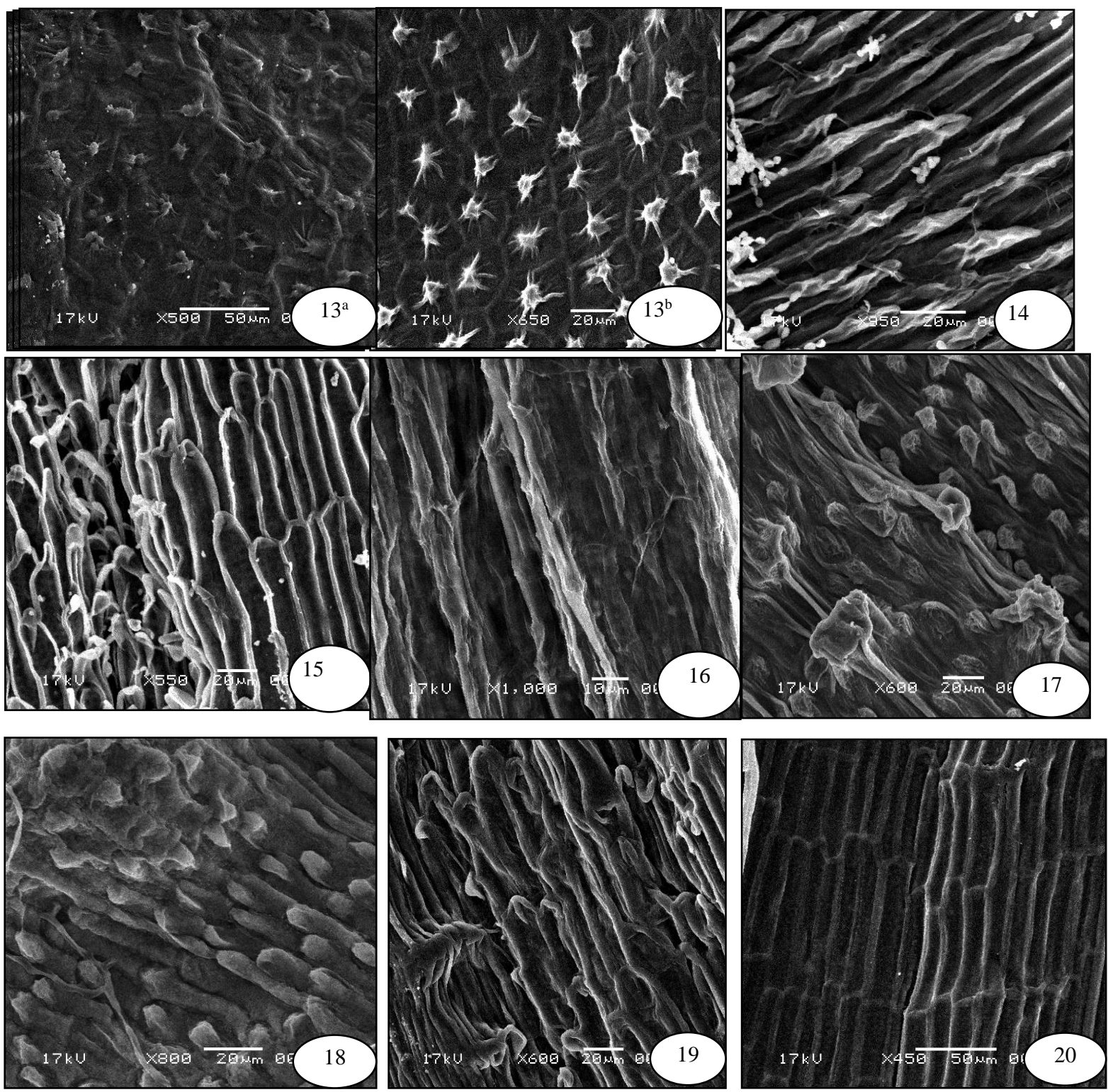

Plate 3con.. Achene surface sculpture for 20 taxa of Asteraceae as revealed by SEM: 13. Calendula arvensis; 14. Urospermum picroides; 15. Launaea spinosa; 16. Launaea nudicaulis; 17.Launaea mucronata sub-sp. mucronata; 18. Reichardia tingitana; 19. Sonchus maritimus; 20. Sonchus oleraceus. 


\section{Seed coat sculpture}

The examination by scanning electron microscope on 20 taxa show differences in achene coat pattern which suggest its grouping into eight groups; the reticulate is the main types in which eight taxa belong to it:

The different types of achene coat pattern are summarized in Table 3 \& plate 3.

1- Reticulate, with two subtypes

a- Regular reticulate; eg. Calendula arvensis (Plate 3, Fig. 13)

b- Reticulate-rugose;eg Sonchus oleraceus (Plate 3 and Fig. 20)

2- Tuberculate achene coat recorded in four taxa; eg. Senecio vulgaris (Plate 3, Fig. 12)

3- Sulcate achene coat found in two taxa; eg. Centaurea aegyptiaca (Plate 3, Fig.4)

4- Puncticulate achene coat for Carduus pycnocephalus (Plate 3 Fig.1)

5- Colliculate achene coat for Notobasis syriaca (Plate 3, Fig. 2)

6- Aculeate achene coat for Pulicaria undulate (Plate 3, Fig. 9)

7- Lineate achene coat for Conyza bonariensis (Plate 3, Fig. 6)

8- Striate achene coat for Pluchea dioscoridis (Plate 3, Fig. 5)

The key (1): The recorded data table $2 \& 3$ were used to construct the following indented key to the 20 taxa of Asteraceae that it might help in the confirmation of their identification.

A- Achene polymorphic, spiny, curved, pappus absent and periclincal wall texture papillate Calendula arvensis AA- Achene homomorphic, not spiny, pappus present and periclincal wall texture not papillate.

B- Achene shape oblong or oblong obovate

C- Achene shape oblong

D- Achene colour brown

E- Achene size $0.5 \mathrm{~mm}$. with granulates texture...........Pseudognaphalium lutedalbum

EE- Achene size more than $0.5 \mathrm{~mm}$. and texture nott granulate

F- Achene texture smooth

G- Ridges absent, pappus type scabrous subulate scales free and achene coat pattern reticulate-rugose

Silybum marianum var. albiflorum
GG- Ridges present, pappus type scabrous barbellate bristles and achene coat pattern striate. Pluchea dioscoridis FF- Achene texture sparsely hairy

$\mathrm{H}$ - Pappus capillary barbellate with limited number \& connate at the base and achene coat pattern reticulate-rugose

Phagnalon rupestre

$\mathrm{HH}$ - Pappus scabrous subulate scales free with unlimited number \& not connate at the base and achene coat pattern sulcate DD- Achene colour not brown Centaurea aegyptiaca

I- Ridges present, stylopodium conspicuous, pappous deciduous with unlimited number and achene coat pattern puncticulate or reticulate

J- Pappus type scabrous subulate scales free with yellowish brown colour and achene coat pattern puncticulate.

\section{Carduus pycnocephalus}

JJ-Pappus type scabrous barbellate bristles with white colour and achene coat pattern reticulate Sonchus maritimus

II- Ridges absent, stylopodium inconspicuous, pappous persistent with limited number and achene coat pattern lineate.

\section{Conyza bonariensis}

CC- Achene shape oblong obovate

$\mathrm{K}$ - Beak and ridges present with tuberculate texture, pappus homogenous and achene coat pattern reticulate

\section{Urospermum picroides}

KK- Beak and ridges absent with sparsely hairy texture, pappus hetrogenous and achene coat pattern aculeate

Pulicaria undulate

BB- Achene shape obovate, fusiform or tetragonal

L- Achene shape obovate

$\mathrm{M}$ - Achene texture smooth

$\mathrm{N}$ - Ridges absent, pappus sub-terminal, deciduous with plumose bristles type and achen coat pattern colliculate .... Notobasis syriaca NN- Ridges present, pappus terminal, persistent with scabrous barbellate fine bristles type achene coat pattern sulcate.

\section{Launaea nudicaulis}

MM- Achene texture tuberculate

O- Pappus yellow, limited with paleaceous scales corona type

...Anthemis melampodina subsp. Deserti 
OO- Pappus white, unlimited with scabrous barbellate fine bristles type............... Launaea spinosa

LL- Achene shape fusiform or tetragonal

$P$ - Achene shape fusiform and pappus type scabrous barbellate fine bristles

Q- Achene colour brown with tuberculate texture and ridges present

$R$ - Pappus perisistent and achene coat pattern tuberculate

\section{..........................Launaea mucronata}

sub-sp. mucronata

RR- Pappus deciduous and achene coat pattern reticulate-rugose...Sonchus oleraceus

QQ- Achene colour green or brownish green with hairy texture and ridges absent

S- Achene colour green.... Senecio vulgaris

SS- Achene colour brownish green......... Senecio glaucus sub-sp. coronopifolius PP- Achene shape tetragonal and pappus type scabrous barbellate bristles......... Reichardia tingitana

The key (2):

Characters: 22 indata, 22 included, 7 in key. Items: 20 indata, 20 included, 20 in key.

Parameters: Rbase $=1.40$ Abase $=2.00 \mathrm{Re}$ use $=1.01$ Varywt $=0.80$

Charactersincluded: 1-22

Character reliabilities: $1-22,5$

1. Coat pattern sculpture puncticulate...... Carduus pycnoephalus

Coat pattern sculpture colliculate.

Notobasis syriaca

Coat pattern sculpture reticulate- rugose.....2

Coat pattern sculpture sulcate................ 4

Coat pattern sculpture aculeate.....

Pulicaria undulate

Coat pattern sculpture lineate.

Conyza bonariensis

Coat pattern sculpture striate.

Pluchea dioscoridis

Coat pattern sculpture tuberculate. $\ldots 5$

Coat pattern sculpture reticulate.......... Caendula arvensis

2(1). Pappus type scarbous barbellatefine bristles... ... 3

Pappus type scarbous barbellate bristles

Pseudognaphalium Iutedalbum

Pappus type scabrous subulate scales free Silybum marianum

Pappus type Paleaceous scales to form corona.. Anthemis melampodina subsp. deserti
Pappus type Plumose bristles.

Urospermum picroides

Pappus type Caillary barbellate. ...

Phagnalonrupestre

3 (2). Seed shape oblong....

Sonchus maritimus

Seed shape obovate.

\section{Launaea spinosa}

Seed shape fusiform

4(1). Seed shape oblong; Pappus type scabrous subulate scales free; Seed color brown; Seed texture sparsely hairy

Centaurea aegyptiaca

Seed shape obovate; Pappus type scarbous barbellatefine bristles; Seed color green; Seed texture smooth....Launaea nudicaulis 5(1). Seed color brown.................. 6 Seed color green.............Senecio vulgaris

Seed color brownish green............... Senecio glaucus sub-sp. coronopifolius 6(5). Seed shape fusiform; Pappus type scarbous barbellatefine bristles; Seed ridges present; Seed stylopodium conspicuous .....Launaea mucronata sub-sp. mucronata Seed shape tetragonal; Pappus type scarbous barbellate bristles; Seed ridges absent; Seed stylopodium inconspicuous.

\section{Reichardia tingitana}

To conclude, achene micro-morphological characters are important and could be helpful in the identification species and genera. Studies on the other taxa of this tribe may further invoke that micro-morphological characters of achene are truly valuable characters to classify the species.

\section{REFERENCES}

Abd El-Twab, M.H. and Zahran F.A. 2010. RAPD and RELP analysis of phylogenetic relationships among congeneric species (Anthemideae, Asteraceae) in Egypt. Int. J. Bot., 6(1): 1-10.

Abd El-Wahid, A., Soliman, M.I., Riz R.M., and Rizk R.M. 2009. Cytogenetical Studies on Achene Colour Polymorphism of Picris asplenoides L. and Urospermum picroides L. (Asteraceae) in Egypt. Pak. J. B. S. 12(7): 565-573.

Amin, A. 1978. Taxonomic revision of the genus Launaea (Compositae). Taeckholmia, 9: 111-117. 
Baldwin, B.G., Wessa, B.L. and Panero, J.L. 2002. Nuclear rDNA evidence for major lineages of Helenioid Heliantheae (Compositae). Systematic Botany, 27: 161-198.

Bednorz, L. and Podsiedlik, M. 2013. A study on achene macro- and micromorphological characters of polish species of the Senecio jacobaea group. ACTA AGROBOTANICA, 66(2): 13-20.

Bhar, I. and Mukherjee, S.K. 2004. Macromorphological and micromorphological study of cypselas in seven species of the tribe Anthemideae (Asteraceae). J. Econ. Taxon. Bot. 28(3): 788-794.

Bisby, F.A., Roskov, Y.R., Orrell, T.M., Nicolson, D., Paglinawan, L.E., Bailly, N., Kirk, P.M., Bourgoin T. and Baillargeon, G. 2010. Species 2000 \& ITIS Catalogue of Life: 2010 Annual Checklist. CDROM; Species 2000: Reading, U.K.

Boulos L. 2002. Flora of Egypt, vol. 3 (Verbenaceae-Compositae). Al Hadara Publishing, Cairo, Egypt, 373 pp.

Bremer, K. 1994. Asteraceae. Cladistics \& Classification. Timber Press, Portland (Oregan). 752 pp.

Bremer, K. and Jansen R.K. 1992. A new subfamily of the Asteraceae. Annals of the Missouri Botanical Garden, 79: 414415.

Cassini, 1827. Cassini H: Saussuress. In: Cuvier, G. (Ed.). Dictionriaire des Sciences Naturelles. 1827; 47: 494 - 513. Paris: Le Normant.

Chehregani, A. and Mahanfar N. 2007. Achene micro-morphology of Anthemis (Asteraceae) and its allies in Iran with emphasis on systematic. Int. J. Agri. Biol., (3): 486-488.

Cronquist, A. 1977. The Compositae revisited, Brittoniq. 29 (2): 137-153.

Cronquist, A. 1955. Phylogeny and taxonomy of the Compositae. American Midland Naturalist 53: 478-511.

Dallwitz, M.J., Paine T.A. and Zurcher E.J. 2000. User's guide to the DELTA system: A general system for processing taxonomic descriptions. 4.12 ed. CSIRO Division of Entomology, Canberra, ACT, Australia. [also available at: http://deltaintkey.com].
Davis, P.H. 1975. Flora of Turkey and the east Aegean islands. Edinburgh University Press, Edinburgh, 5: 173-324.

El-Karemy, Z.R. and Zareh, M. 1991. Systematic revision of Compositae in Egypt. 8. tribe Cardueae: Carduus and allied genera. Mitt. Bot. München, 30: 439-57.

Fayed, A.A. and Zareh M. 1987. Systematic revision of Compositae in Egypt. 2. Tribe Senecioneae Cass. Taeckholmia, 10: 6775.

Fayed, A.A. and Zareh M. 1989. Systematic revision of Compositae in Egypt. 4. Tribe Inuleae Gnaplalium and related genera. Willdenowia, 18: 445-53.

Fayed, A.A. and Mohamed M. 1991a. Systematic revision of Compositae in Egypt. 5. Tribe Inuleae: Pulicaria and related genera. Willdenowia, 20: 81-89.

Fayed, A.A. and Mohamed M. 1991b. Systematic revision of Compositae in Egypt. 6. Tribe Inuleae: Inula and related genera. Willdenowia, 20: 91-96.

Fayed, A.A. and Zareh M. 1988. Systematic revision of Compositae in Egypt. 3. Tribe Inuleae: Filago and Ifloga. Willdenowia, 17: 115-23.

Fayed, A.A. 1987. Systematic revision of Compositae in Egypt. 1. Tribe. Astereae Cass. Taeckholmia, 10: 1-11.

Fayed, A.A. 1991. Systematic revision of Compositae in Egypt. 7. Tribe Inuleae: Phagnalon and Leysera. Willdenowia, 20: 97-102.

Hickey, M. and King C. 1997. Common Families of Flowering Plants. Cambridge University Press, U.K.

Jana B.K. and Mukherjee S.K. 2012. Diversity of cypselar features of seven species of the genus crepis L. in compositae, Indian Journal of Fundamental and Applied Life Sciences, 2(1): 51-58.

Jana, B.K. and Mukherjee S.K. 2013. Cypselar diversity between two taxa of the tribe madieae (Compositae). Indian Journal of Plant Sciences. 2(3): 23-27.

Jana B.K. and Mukherjee S.K. 2014. Diversity of testal structure among some tribes of Compositae. Journal of Science, 4(5): 327-338.

Jana B.K., Bar, R. and Mukherjee S.K. 2013. Cypselar morphology and anatomy of five species of the tribe InuleaeAster- 
aceae. International Journal of Pharma and Bio Sciences, 4(1): 911-919.

Mukherjee, S.K. and Nordenstam B. 2008. Diversity of pappus structure in sometribes of the Asteraceae. Phytotaxonomy, 8: 32 - 46.

Osman, A.K. 2011a. Pollen Morphology of Tribes Gnaphalieae, Helenieae, Plucheeae and Senecioneae (Subfamily Asteroideae) of Compositae from Egypt. American Journal of Plant Sciences, 2: 120133.

Osman, A.K. 2011b. Numerical taxonomic study of some tribes of Compositae (subfamily Asteroideae) from Egypt. Pak. J. Bot., 43(1): 171-180.

Panero, J. L. and Funk V.A. 2002. Toward a phylogenetic subfamilial classificastion for the Compositae (Asteraceae). Proceedings of the Biological Society of Washington, 115: 909-922.

Täckholm, V. 1974. Students' Flora of Egypt. ed. 2. Cairo Univ. Egypt.
Takhtajan, A. 1997. Dirersity and Classification of Flowering Plants. New York: Columbia University Press.

Talukdar, T. and Mukherjee S.K. 2008. Comparative study of cypselas in three common species of Asteraceae. Pleione. 2(1): 147-149.

Thorne, R.F. 1983. Proposed new realignments in the Angiosperms. Nordic. .J. Bot. 3: 85-117.

Zareh, M. and Osman A. 2004. Systematic and palynological studies on Heliantheae (Asteraceae) in Egypt. Bull. Fac. Sci., Assiut Univ., 33: 197-208.

Zareh, M.M. 2005. Synopsis of the Family Asteraceae in Egypt. Int. J. Agri. Biol., 7(5): 832-844.

Zareh, M. 1987. Taxonomic and achene anatomy of species of Anthemideae (Asteraceae) in Egypt. Unpublished Ph.D. Thesis; Assiut. Univ., Assiut. Egypt.

Zareh, M. 1992. Systematic revision of Compositae in Egypt. 9. Tribe Cardueae: The genus Atractylis L. Bull. Fac. Sci., Assiut Univ., 21: 1-11. 